\title{
An Efficient Segmentation Method for Overlapping Chromosome Images
}

\author{
Tanvi \\ Research Scholar \\ Department of Computer Science \& Engineering, \\ National Institute of Technology \\ Jalandhar, Punjab, India
}

\author{
Renu Dhir, Ph.D \\ Associate Professor and HOD of Computer \\ Science \& Engineering, \\ National Institute of Technology, \\ Jalandhar, Punjab, India
}

\begin{abstract}
Systems are being developed to automate the task of classification of chromosomes. The chromosomes are nonrigid material and they are several times touching each other or they overlap each other in the metaphase images. So different techniques are required to segregate the overlapping chromosomes. This paper presents a novel method for segmenting chromosomes based upon computational geometry. In the proposed approach first the contour line is traced for the overlapping chromosomes and then all the cut points are traced for the overlapping chromosomes. Then based on computational geometry method a specific number of cut points are selected and they are used for separating the two chromosomes. We have found that $87.4 \%$ of the images were correctly segmented using the proposed method.
\end{abstract}

\section{Keywords}

Chromosome segmentation, chromosome analysis, overlapping chromosomes

\section{INTRODUCTION}

Chromosomes are the cell structures that carry the genetic details . they are made up of DNA (Deoxyribonucleic acid) and some type of proteins. The DNA contains the unique genetic details and the proteins take care of the DNA and helps so that the DNA duplicates well when the cell division takes place.

Chromosomes are very small in size they can be viewed only with the help of a microscope. The chromosomes are imaged using a microscope during the cell division phase. As otherwise the chromosomes are very long and thin structures. But during the cell division phase they shrink in size and become much shorter and thicker in size. So they become visible to a microscope.

A normal human being has 46 chromosomes in his cell. These 46 chromosomes are paired, so there are 22 pairs comprising of one chromosome from mother and other from the father. In addition to these 22 pairs there are 2 sex chromosomes, in case of females there are two $\mathrm{X}$ chromosomes and in case of males there is one $\mathrm{X}$ and another $\mathrm{Y}$ chromosome. Karyotyping is a technique that is used to represent these 46 chromosomes in pairs according to their decreasing order.

Segregation of overlapping chromosomes is one of the vital step in automatic classification of chromosomes. The procedures for automatic segregation of overlapping chromosomes aim at applying thresholding based methods to divide the different overlapping chromosomes into different chromosomes by using the geometric shapes [6-8], curvature of the boundary of the chromosome $[9,10]$, layout of the chromosome [11] and pattern of the bands [12,13] in this paper an attempt has been made to propose a efficient segmentation method for overlapping chromosomes using computational geometry based approaches that have been successfully used for various image analysis tasks [14-16]

\section{LITERATURE SURVEY}

There has been extensive research from the last 40 years to develop a mechanism to automatically segment the chromosome images so as to diagnose the chromosomes and find out if any anomaly exists in the chromosome. The automatic analysis of the chromosome images is a six step process. The steps can be listed as:

- $\quad$ Preprocessing

- Segmentation

- Feature Extraction

- Feature Selection

- Classification

- Outlier's detection/ Anomaly detection

The preprocessing phase aims at removing the noise, improving contrast and enhancing the edges. The segmentation phase aims at differentiating chromosomes from the background and other biological material present in the image. The feature extraction phase measures the features such as size, shape, area, band patters, centromeric index, density profile etc. After the feature extraction phase the most discriminative features are selected so as to be used for the classification phase. The classification phase classify the segmented chromosomes into the 24 classes based upon the selected features. Once the chromosomes have been classified they can be analyzed for abnormalities based upon outliers.

The image segmentation methods are mostly based upon the edge detection, thresholding, region based segmentation or upon watershed transform. All these methods work fine when the images are clear, but these methods fail while segmenting images that are partially overlapped. Most of the literature available use manually segmented databases for chromosome classification or they are semi automatic in nature that takes human interventation during the segmentation of overlapping chromosomes.

Liang [8] has given an efficient technique for chromosome segmentation taking into consideration the concave shape of the chromosomes, he has used heuristics search to find out minimum density edges between touching chromosomes. But this method is dependent upon images taken from a single laboratory, the procedure is very time consuming and is dependent upon the origin of the split line . they have not considered the overlapping chromosomes.

Boaz Lerner [26] has proposed a classification driven segmentation approach based upon mapping based upon principal component approach for feature selection and have 
used multilayer preceptron for classification. This approach is automatic and it considers overlapping and touching chromosomes. But they have considered only those images that have only one cluster of touching chromosomes. This method works well for normal chromosomes but they have not mentioned anything about abnormal chromosomes.

Agam[9] has worked on grayscale chromosome images and has used miniset based approach to disassociate touching and overlapping chromosomes. The work is based upon hypothesis of shape testing to find cut points that can potentially divide the chromosomes in a cluster. The method was successful in many aspects but was limited to grayscale images.

Graham [27] has used a technique based upon split and merge and water sheds to over segment the images. The proposed method is same as the method of region growing that grow the segments until they touch. This method is suitable for touching chromosomes. But this method cannot handle overlapping chromosomes.

L. Vanderheydt [28] they have used fuzzy based set theory for segmentation of chromosomes. For the high curvature points of boundary they have defined fuzzy relations. The boundaries have been defined using fuzzy subsets. This method is a very good approach for simple touching and overlapping images but fails when segmenting highly clustered and bent chromosomes.

Vossepoel [29] has tried to define rules using which they can calculate potential cut points and then use an algorithm to link those cut points. This method can find the chromosome boundaries but it cannot handle the overlapping chromosomes.

Q. Wu [30] has proposed a method based upon a model that depicted boundary features based upon touching and overlapping chromosomes. This technique was capable of separating the chromosomes from clusters but it had a rapid rate of failure.

Ji[31] has worked upon grey scale images using geometric information, they have been quite successful to segment and classify the images by using convex hulls to remove overlaps. But this method works for grey scale images.

Lijiya [32] has proposed a scheme based upon majority voting for segmentation and classification of human chromosomes images. They have used fuzzy logic classifier for the classification purpose. The proposed technique is not very efficient and is not applicable to touching and overlapping chromosomes.

\section{PROPOSED METHOD}

Binarization helps to distinguish between the fore ground and back ground pixels. It is one of the critical step in image preprocessing. One of the most basic and simplest method for binarization is global thresholding. The global thresholding technique divides the pixels into fore ground and back ground pixels. It is based upon histogram analysis.

$G(x, y)=\left\{\begin{array}{ccc}1 & & \\ 1 & \text { if } & f(x, y)>T \\ 0 & \text { if } & f(x, y)<=T\end{array}\right\} \rightarrow E q 1$
Thresholding assigns a value of 0 or 1 based upon a global value of thresholding $\mathrm{T}$ based upon the equation 1 . The threshold values are selected from the histograms of the images.

The below figure shows the binarized image of the overlapping chromosomes by applying the global thresholding method.
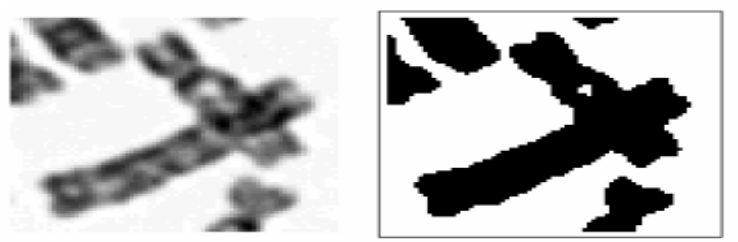

\section{Overlapping}

Chromosomes (b) chromosomes after thresholding

After thresholding he image, the contours can be found for the image. The contours are the foreground pixels that touch the background pixels. These contours can be obtained by using the convolution of matrix $\mathrm{C}$ and function $\mathrm{T}$ given by equation $2 \& 3$ respectively.

$$
\begin{aligned}
& C=\left[\begin{array}{ccc}
0 & 0.25 & 0 \\
0.25 & 1 & 0.25 \\
0 & 0.25 & 0
\end{array}\right] \rightarrow E q 2 \\
& T=(x, y)=\left\{\begin{array}{c}
1, \text { if } 1<f(x, y)<2 \\
0, \text { if } 1>=f(x, y)<=2
\end{array}\right\} \rightarrow E q 3
\end{aligned}
$$

Further the following curvature function can be used for interpolation.

$R=\frac{\partial \phi(s)}{\partial s} \equiv \frac{1}{p}=\frac{\ddot{y}}{\left(y^{2}+1\right)^{3 / 2}} \rightarrow E q 4$

The curvature can be calculated by finding out the difference between the slope at the pixel and the slope at its left neighborhood.

The following image shows the overlapping chromosome image (a) after thresholding, (b) after convolution and (c) contour image.

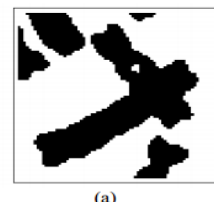

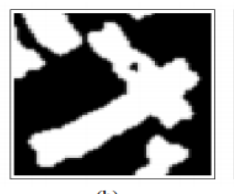

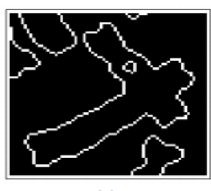

After finding the counters and curvature the possible cut points are detected. The contour pixels where there is sudden change in intensity. So from curvature function the cut points are found. 
After finding out the possible cut points the varonoi diagrams can be used to find out center of the overlapping area. From varonoi diagram the overlapping portion can be obtained.

After this the overlapping chromosomes can be segregated by using the cut points and the contour set.

\section{RESULTS AND DISCUSSION}

The proposed algorithm was applied on the overlapping chromosome images obtained from BioImlab's website. Its dataset named chromosome images for segmentation was used to test and validate the algorithm. The dataset contains 162 images.

For the above dataset following results were obtained:

\begin{tabular}{|l|l|l|}
\hline $\begin{array}{l}\text { Correctly } \\
\text { Segmented }\end{array}$ & $\begin{array}{l}\text { Incorrectly } \\
\text { separated }\end{array}$ & $\begin{array}{l}\text { Cannot be } \\
\text { separated }\end{array}$ \\
\hline $87.4 \%$ & $5.3 \%$ & $7.3 \%$ \\
\hline
\end{tabular}

The results are quite encouraging as compared to other methods. The algorithm can be further improved by considering more cut points so as to get better precision in finding out the overlapping chromosomes and thus segmenting them more accurately. The chromosomes that cannot be separated are mainly highly cluttered.

\section{REFERENCES}

[1] A Carothers and J Piper. Computer-aided classification of human chromosomes: A review. Statist. Computing 1994; $4,161-71$.

[2] B Lerner. Toward a completely automatic neural-networkbased human chromosome analysis. IEEE Trans. Syst. Man Cybern. B: Cybern. 1998; 28, 544-52.

[3] FCA Groen, TK Kate, AWM Smeulders and IT Young. Human chromosome classification based on local band descriptors. Patt. Recog.Lett. 1989; 9, 211-22.

[4] J Piper, E Granum, D Rutovitz and H Ruttledge. Automation of chromosome analysis. Sig. Proc. 1980; 2 , 203-21.

[5] M Moradi and SK Setarehdan. New features for automatic classification of human chromosomes: A feasibility study. Patt. Recog. Lett. 2006; 27, 19-28.

[6] J Liang. Intelligent splitting in the chromosome domain. Patt. Recog. 1989a; 22, 519-32.

[7] J Liang. Decomposition of overlapping chromosomes. In: C Lundsteen and J Piper (ed). Automation of Cytogenetics, Springer, Berlin, 1989b, p. 177-90.

[8] J Liang. Fully automatic chromosome segmentation. Cytometry 1994; 17, 196-208.

[9] G Agam and I Dinstein. Geometric separation of partially overlapping nonrigid objects applied to automatic chromosome classification. IEEE Trans. Patt. Anal. Mach. Intell. 1997; 19, 1212-22.

[10] B Lerner, H Guterman and I Dinstein. A classificationdriven partially occluded object segmentation (CPOOS) method with application to chromosome analysis. IEEE Trans. Sig. Proc. 1998; 46, 2841-47.
[11] M Popescu, P Gader, J Keller, C Klein, J Stanley and C Caldwell. Automatic karyotyping of metaphase cells with overlapping chromosomes. Comp. Biol. Med. 1999; 29, 61-82.

[12] GC Charters and J Graham. Trainable grey level models for disentangling overlapping chromosomes. Patt. Recog. 1999; 32, 1335-49.

[13] GC Charters and J Graham. J. Disentangling chromosome overlaps by combining trainable shape models with classification evidence. IEEE Trans. Sig. Proc. 2002; 50, 2080-85

[14] R Ogniewicz and M Ilg. Voronoi skeletons: Theory and applications. In: Proceedings of the IEEE Conference on Computer Vision and Pattern Recognition, Illinois. 1992, p. 63-9.

[15] M Styner, G Gerig, J Lieberman, D Jones and D Weinberger. Statistical shape analysis of neuroanatomical structures based on medial models. Med. Image Anal. 2003; 7, 207-20.

[16] CM Boesse, MK Henry, TW Hyde and LS Matthews. Digital imaging and analysis of dusty plasmas. Adv. Space Res. 2004; 34, 2374-78.

[17] PK Sahoo, S Soltani and AKC Wong. A survey of thresholding techniques. Comp. Vis. Graph. Image Proc. $1988 ; 41,233-60$

[18] RM Haralick and LG Shapiro. Computer and Robot Vision, Vol. I, Addison-Wesley, Reading, MA, 1992, p. $1-608$

[19] N Otsu. A threshold selection method from gray-level histograms. IEEE Trans. Syst. Man Cybern. 1979; 9, 62 6.

[20] S Wolfram. The Mathematica Book, 5th ed., Book News, Oregon, 2004, p. 1-1200.

[21] H Freeman and LS Davis. A Corner Finding Algorithm for Chain Coded Curves. IEEE Trans. Comp. 1977; 26 , 297-303.

[22] T Pavlidis. Algorithms for Shape Analysis of Contours and Waveforms. IEEE Trans. Patt. Anal. Mach. Intell. $1980 ; 2,301-12$.

[23] A Rosenfeld and AC Kak. Digital Picture Processing. Academic Press, California, 1982, p. 1-349.

[24] W Srisang, K Jaroensutasinee and M Jaroensutasinee. Segmentation of overlapping chromosome images. In: Abstracts of the 30th Congress on Science and Technology of Thailand, Bangkok, Thailand. 2004, p. 40.

[25] E. Grisan, E. Poletti, A. Ruggeri. Automatic segmentation and disentangling of chromosome in Q band prometaphase images, IEEE Trans Inf Technol B, 2009.

[26] Boaz Lerner: Toward a completely automatic neuralnetwork-based human chromosome analysis. IEEE Transactions on Systems, Man, and Cybernetics, Part B 28(4): 544-552 (1998)

[27] J. Graham, "Resolution of Composites in interactive karyotyping", in automation of cytogynetics,191-203, Springer-Verlag, Berlin,1989. 
[28] L. Vanderheydt, F. Dom, A. Oosterlinck, and H. Van Den Berghe, "Two-Dimensional Shape Decomposition Using Fuzzy Subset Theory Applied to Automated Chromosome Analysis," Pattern Recognition, vol. 13, no. 2, pp. 147-157, 1981.

[29] A.M. Vossepoel, Analysis of Image Segmentation for Automated Chromosome Identification, University of Leiden, Leiden,Netherlands, Doctoral Dissertation, 1987. [30] Q. Wu, Automated Identification of Human Chromosomes as an Exercise in Building Intelligent Image Recognition Systems, Catholic University of
Leuven, Leuven, Belgium, Doctoral Dissertation, 1987.

[31] L. Ji, "Intelligent Splitting in the Chromosome Domain," Pattern Recognition, vol. 22, no. 5, pp. 519532,1989

[32] Lijiya, A, K.S.Sreejini, V.K.Govindan. 2012. M-FISH Chromosome Images Classification by Watershed based Segmentation Approach, 25 March,2012. International conference on advances in Computer,Electrical and Electronics Engineering(ICACEEE-2012), Bombay,India 